Revue musicale OICRM

\title{
Présentation du numéro " La profession musicale au Québec »
}

\section{Michel Duchesneau et Danick Trottier}

Volume 1, numéro 1, 2012

Profession musicale au Québec

URI : https://id.erudit.org/iderudit/1055854ar

DOI : https://doi.org/10.7202/1055854ar

Aller au sommaire du numéro

Éditeur(s)

OICRM

ISSN

2368-7061 (numérique)

Découvrir la revue

Citer ce document

Duchesneau, M. \& Trottier, D. (2012). Présentation du numéro « La profession musicale au Québec ». Revue musicale OICRM, 1(1), i-viii.

https://doi.org/10.7202/1055854ar d'utilisation que vous pouvez consulter en ligne.

https://apropos.erudit.org/fr/usagers/politique-dutilisation/ 


\title{
revue musicale oicrm
}

Le site de la Revue musicale de l'Observatoire interdisciplinaire de création et recherche en musique

\section{Présentation du numéro \\ "La profession musicale au Québec », vol. $1, \mathrm{n}^{\circ} 1(2012)$}

\author{
Michel Duchesneau ${ }^{1}$ \\ et Danick Trottier ${ }^{2}$
}

ÉDITORIAL

Lancer une nouvelle revue scientifique est en soi un défi. Un tel projet fait surtout face à une contrainte importante, liée à la multiplication des supports de diffusion. Pourquoi donc une nouvelle revue consacrée à la musique ? Cette question trouve sa réponse dans les réalités scientifiques et culturelles du Xxi ${ }^{\mathrm{e}}$ siècle, lesquelles nous invitent à tenir compte du contexte de diffusion de la recherche et des plateformes appropriées. En ce sens, la Revue musicale OICRM (RMO) fait le pari que la recherche scientifique doit aussi prendre le virage de l'ère numérique. C'est dans ce contexte que nous avons voulu offrir à la communauté scientifique musicale un nouvel outil de diffusion et une nouvelle plateforme interactive, la RMO étant une revue francophone du web qui publie des articles en français et en anglais. En effet, le souhait qui accompagne la création de la revue est de favoriser la publication de textes qui exploitent au mieux les possibilités multimédia offertes par la plateforme informatique du web.

Cette revue, de par son format et son contenu, cherche à refléter la richesse des disciplines et des perspectives de recherche menées dans le domaine de la science musicale, et dont l'Observatoire interdisciplinaire de création et de recherche en musique (OICRM), qui finance le projet, témoigne à travers ses différents laboratoires. La RMO est ouverte aux domaines de la musicologie, de la composition ou de la pratique instrumentale avec toutes les sous-disciplines impliquées au sein de ces trois univers. Et surtout, elle souhaite stimuler les articles ayant une dimension pluridisciplinaire ou relevant d'une collaboration entre plusieurs chercheurs. Dans ce contexte, et dans la perspective où le médium choisi mérite d'être exploité à travers toute sa richesse, la revue vise à encourager la soumission d'articles qui intégreront des documents multimédia, que ce soit sous la forme de fichiers vidéo de performances musicales, de fichiers audio, de tableaux, etc. Au-delà de leur dimension illustrative, ces documents auront également vocation à consolider l'argumentaire et l'analyse proposés par les auteurs.

De ce point de vue, la RMO est une invitation à repenser la façon de développer et de transmettre les connaissances sur les musiques par le biais d'outils qui élargissent les possibilités habituellement offertes par une revue imprimée. Mais nous nous faisons aussi un devoir d'accueillir des articles écrits

1 Professeur titulaire, Université de Montréal et Directeur, OICRM.

2 Chargé de cours, Université de Montréal et Agent de recherche, OICRM. 
dans un format classique, sans contenu numérique. Aussi, l'arrivée d'une nouvelle revue coïncide fréquemment avec la mise en chantier de nouveaux projets et de nouveaux noms. En ce sens, nous offrons la possibilité à de jeunes chercheurs de publier leurs premières recherches. Aussi, le rapport de la recherche sur la musique à son environnement immédiat est au cœur de nos préoccupations. C'est pourquoi la revue proposera des «dossiers de recherche » qui créeront des liens étroits entre l'activité de recherche fondamentale et le milieu musical, pédagogique, institutionnel et professionnel.

La RMO sera constituée de deux numéros par année, chaque numéro comportant deux volets : un dossier, suivi d'une section libre. Nous pouvons d'ores et déjà annoncer qu'après ce premier numéro sur la professionnalisation musicale au Québec, les prochains porteront sur la réception de Debussy et sur une problématique en esthétique. Mais nous tenons à préciser qu'à tout moment un projet d'article peut être soumis aux rédacteurs de la revue. Vous pouvez obtenir de plus amples renseignements sur le site Internet de la revue et y trouver aussi le protocole de rédaction.

La RMO compte sur un comité scientifique dont les membres sont internationalement reconnus au sein de leurs disciplines respectives. Nous tenons à remercier les chercheurs et professeurs qui ont accepté notre invitation. Nous voulons aussi leur exprimer notre gratitude pour le travail accompli dans le cadre de ce premier numéro. Enfin, il nous sera aussi permis de remercier plusieurs personnes qui ont été indispensables dans l'organisation et la planification de ce numéro. Michel Duchesneau, directeur, et Serge Lacasse, co-directeur de l'OICRM nous ont accompagnés durant le processus de gestation du projet scientifique et ont activement contribué à la qualité de la revue de par leurs commentaires constructifs. L'aide d'Ariane Couture aura aussi été précieuse, notamment dans la mise en ligne des articles figurant dans le présent numéro. Quant au projet lui-même, il n'aurait pu voir le jour sans l'engagement programmatique de Yannick Bélanger et Sophie Lavoie d'ID Fix, auxquels nous exprimons de chaleureux remerciements. Enfin, ce numéro n'aurait pu voir le jour sans l'enthousiasme de celles et ceux qui ont répondu favorablement à notre appel à publication. Longue vie à la revue!

Le rédacteurs de la revue : Nathalie Fernando, Frédéric Léotar et Danick Trottier

Ce premier numéro de la Revue musicale OICRM (RMO), dont le dossier porte sur la professionnalisation en musique du Québec, est à l'image de l'esprit qui animera cette revue au cours des années à venir. D'une part, sans être une revue étudiante, la Revue musicale OICRM fait une large place aux travaux des étudiants qui soumettent des articles répondant aux objectifs scientifiques de la revue. Cette réalité s'inscrit dans l'importance des travaux des étudiants au sein de la communauté universitaire, tant à l'Université de Montréal et l'Université Laval

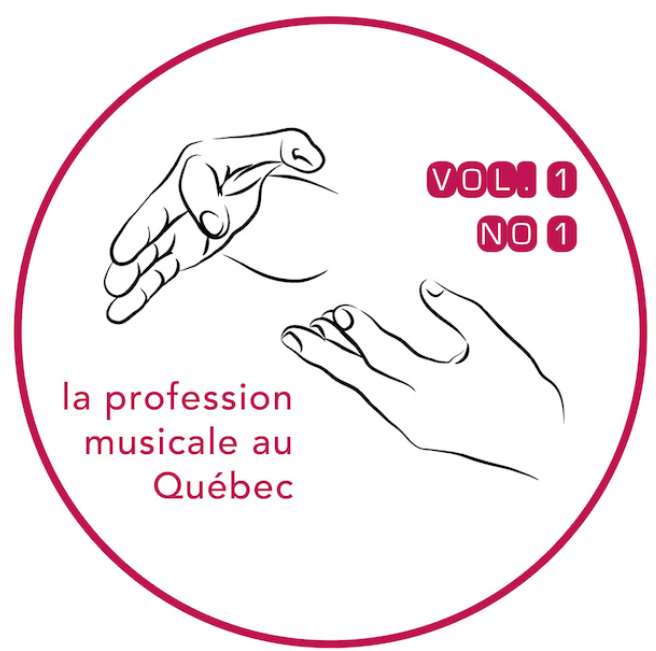
(piliers de l'OICRM) que dans les autres universités francophones. C'est dans cette optique que sont présentés aujourd'hui trois articles rédigés par des étudiants qui ont suivi un séminaire sur la " professionnalisation en musique » en mai et juin 2011, deux articles issus des travaux sur la pédagogie musicale sous la direction de Francis Dubé et un article sur le travail stylistique de la voix dans le country-western québécois des années 1930 et 1940. D'autre part, ce numéro montre aussi l'originalité et l'ouverture aux différents domaines disciplinaires qui alimentent la recherche sur la musique. 
Dans ce premier numéro, la sociomusicologie, la pédagogie et l'analyse phonostylistique sont à l'honneur, ce qui témoigne du dynamisme que l'on retrouve dans les différents secteurs d'activité de l'OICRM.

Les travaux sur la professionnalisation des musiciens sont relativement nombreux, mais ils concernent des milieux spécifiques comme la France et les États-Unis. À cet égard, on ne saurait faire l'économie de la mise en garde d'Eliot Freidson dans son article devenu célèbre sur le défi que pose l'étude des professions artistiques : "Dans le cas des professions artistiques, la diversité des politiques nationales suscite de grandes différences, d'une part dans les carrières sur le marché du travail, d'autre part dans les définitions sociales du statut d'artiste " (Freidson 1986, p. 431). On aura compris que les travaux réalisés en France sur la profession musicale ne peuvent être appliqués directement au Québec, la situation de la province canadienne étant plutôt à mi-chemin entre le soutien d'État et le libre marché, bref à un mélange de culture française et de culture américaine. Pour ne donner qu'un exemple, le régime des intermittents du spectacle n'a aucune espèce d'équivalence au Québec, ce qui rend le marché de l'emploi musical beaucoup plus près d'un libre marché, bien que l'État soit présent pour supporter financièrement les artistes mais toujours selon une sélection opérée en amont en fonction des programmes de bourses et de subventions.

C'est dans ce contexte qu'il nous a semblé indispensable de questionner notre milieu quant à la professionnalisation musicale, car le dynamisme du milieu artistique québécois, quoique reconnu internationalement, n'a pas encore fait l'objet d'enquêtes approfondies en ce qui a trait à ses caractéristiques et ses distinctions fonctionnelles et institutionnelles. Or, comme le souligne pertinemment Raymonde Moulin, la capacité des artistes à " entrer et à survivre dans le monde de l'art et dans le marché n['est] pas indépendante des facteurs esthétiques, économiques et institutionnels qui déterminent les contextes dans lesquels se situe l'ensemble des acteurs intervenant dans la construction des réputations " (Moulin 1997, p. 336).

Si des travaux existent dans le domaine des arts de la scène comme le théâtre et la danse $\mathrm{e}^{3}$, le domaine de la musique est peu exploré. Or, le rapport que le milieu universitaire québécois entretient avec le milieu professionnel en musique est étroit puisqu'une part importante des interprètes et des compositeurs sont issus du milieu universitaire par l'intermédiaire des Facultés et des départements de musique, ou y travaillent carrément, rejoignant ainsi l'observation qu'avait formulée Freidson (1986, p. 436) en ce qui concerne le fait que la profession artistique s'exerce à la condition d'une occupation principale, la plupart du temps l'enseignement.

3 Sur les professions artistiques, la grande majorité des données et des travaux émanent de l'Observatoire de la culture et des communications du Québec. C'est le cas par exemple d'une étude approfondie sur les danseurs et chorégraphes québécois, qu'on retrouve d'ailleurs sur le site internet de l'Observatoire : www.stat.gouv.qc.ca/statistiques/culture/arts-scene/danseurs-choregraphes/danseurs-choregraphes.pdf. D'autres travaux touchent aux conditions de l'exercice des professions artistiques au Québec, mais souvent à travers une visée historique ou dans un secteur d'activité bien différent de la musique À cet égard, les arts visuels semblent jouir d'une longueur d'avance, chez les sociologues de la culture québécoise notamment. 
D'autre part, il nous semblait important que les étudiants soient sensibilisés aux réalités et aux problématiques d'un milieu professionnel qu'ils n'abordent qu'obliquement et souvent tardivement dans le cours de leurs études. Le séminaire s'adressait tout autant aux étudiants en musicologie, en ethnomusicologie qu'aux étudiants en interprétation et en composition.

Aborder la question de la professionnalisation musicale au Québec relevait aussi d'un souci de confronter un enseignement musical pour les interprètes encore profondément ancré dans une tradition qui met l'accent, presque exclusivement, sur l'enseignement instrumental pour les interprètes et sur l'enseignement de la composition pour les compositeurs. Cette formation semble à plus d'un égard profondément décalée par rapport aux réalités du marché du travail dans le domaine musical. Il semble y manquer des outils pour faire face au marché musical, par exemple l'organisation d'un curriculum vitae, les réalités syndicales propres aux institutions ou la négociation d'un salaire. Ce phénomène n'est pas nouveau, mais ne cesse de grandir. Pierre-Michel Menger affirme d'ailleurs que « la formation sur le tas et l'apprentissage par l'expérience jouent un rôle si décisif que la formation initiale, dans beaucoup de domaines artistiques, agit comme un levier bien imparfait de l'insertion professionnelle » (Menger 2009, p. 7).

Cette remarque lapidaire de Menger, à notre sens, met en cause l'enseignement moderne des arts et plus particulièrement de la musique, alors que les institutions d'enseignement supérieur revendiquent une formation ouvrant les portes au marché du travail.

L'expérience que révèlent les travaux sur le sujet et tout particulièrement les travaux entrepris dans ce numéro de la RMO, semble confirmer cet état de fait. Sans comprendre les mécanismes en jeu dans un milieu hautement compétitif qui s'inscrit dans le cadre économique large du " marché du travail " par l'entremise d'une réflexion plus théorique sur la notion de profession, l'intégration des jeunes artistes semble, à l'évidence, de plus en plus difficile. Comme l'a remarqué Philippe Coulangeon (2004, p. 175) dans le cas de la France, les vertus de la flexibilité quant aux occupations que peut remplir l'interprète ont aussi leurs limites au Québec. D'autant que la plupart des musiciens, de surcroît en musique classique, sont à la recherche d'un emploi stable, la flexibilité étant toujours conçue comme une situation tolérable mais non totalement acceptable.

Tout le problème peut se résumer dans le célèbre adage repris par plusieurs dans l'étude des professions artistiques : " beaucoup d'appelés mais très peu d'élus ", ce qui est aussi vrai au Québec qu'ailleurs. En fait, l'intégration au marché du travail musical est d'autant plus ardue qu'elle exige non seulement une meilleure compréhension des enjeux du métier, mais une adaptabilité aux nouvelles technologies et aux modes de communication devenus des outils indispensables pour le développement d'une carrière de musicien, de compositeur, voire de musicologue ou d'ethnomusicologue. À cet effet, les pages web et les sites internet sous forme de vitrines promotionnelles développés par les musiciens (les interprètes notamment, en chant par exemple) montrent la nécessité de construire une image glamour, et de bâtir une réputation (Becker [1988]2010, p. 47-48) comme vecteur indispensable d'une reconnaissance pour évoluer au sein du monde musical. 
Cette démarche répond aussi à une autre préoccupation, partagée avec les étudiants, qui est celle d'arrimer plus adéquatement une part de l'enseignement universitaire en musique aux réalités contemporaines du milieu musical, sachant qu'il existera toujours un décalage entre les deux mondes. Moulin écrivait en 1992 que dans le domaine des beaux-arts, " la conquête de l'indépendance à l'égard du marché a été magistralement exemplifiée par la carrière de Dubuffet et, aujourd'hui, le mode d'emploi du marché est partie intégrante de l'enseignement des écoles d'art » (Moulin [1992]1997, p. 7). Vingt ans plus tard, force est de constater que la situation n'est pas égale partout et qu'en musique, il persiste un retard considérable en matière de relation avec le milieu professionnel. Les programmes de "gestion de la musique "sont rares ${ }^{4}$. Et si les HEC Montréal proposent, comme plusieurs autres écoles de sciences économiques des programmes de formation en gestion des arts (toute discipline confondue), ces programmes sont rarement suivis par les futurs artistes, mais bien par les futurs gestionnaires.

Dans le cas présent, à la Faculté de musique de l'Université de Montréal, notre objectif se concentre dans le fait d'offrir aux étudiants un certain nombre de cours et de séminaires où ils pourront prendre connaissance des conditions matérielles de la pratique de leur futur métier (le cours de Métier de musicien par exemple), mais aussi où ils pourront réfléchir sur ces conditions, et en concevoir les fondements dans une optique d'un meilleur arrimage entre l'apprentissage et les réalités du métier. Nous pensons que les compétences théoriques réflexives développées par les nouvelles générations pourront sans aucun doute contribuer à créer une autre dynamique que celle qui existe toujours et qui se résume à celle d'une formation à sens unique pour répondre à une définition restreinte du métier de musicien, définition essentiellement académique, qui ne remplit que partiellement les requis du marché du travail. D'ailleurs, cette réalité est aussi rendue nécessaire par la situation du marché musical actuel au Québec où, comme ailleurs, les frontières sont de plus en plus poreuses entre musique classique et musique pop, entre emploi stable et emploi précaire, notamment pour certaines catégories d'instrument comme les cuivres, le chant ou la guitare.

Cela nous amène à réfléchir sur la définition même de ce qu'est un musicien aujourd'hui. Si l'enseignement supérieur de la musique conduit à la figure du "spécialiste ", soit un artiste ayant une formation de pointe qui l'élève à un statut d'élite, on est bien contraint de considérer que cette élite ne sera pas seule à dominer la scène musicale. L'excellence artistique n'est plus la seule garantie de réussite dans un milieu où l'artiste " préfabriqué » ou l'autodidacte occupe une place de plus en plus grande. La formation et le talent sont alors remplacés par l'ingéniosité, le soutien des outils informatiques d'enregistrement ou de création sonore ou encore l'utilisation habile des médias et, malheureusement, trop souvent, le charlatanisme. L'excellence artistique ne garantit pas non plus la possibilité d'établir une réputation durable qui

4 On pense notamment au Master professionnel en administration et gestion de la musique offert par l'universités de Paris-Sorbonne et l'université Jean Monnet à Saint-Étienne ou au diplôme d'université en économie et gestion des projets musicaux donné à l'université Paris-Ouest Nanterre La Défense. 
assure une stabilité à l'artiste, puis lui ouvre les portes du " succès ». Pourtant, ce succès artistique est bien souvent à la base du désir de devenir un artiste ; il constitue à tout le moins un puissant stimulant pour minimiser les risques de l'incertitude liée à la projection de la carrière. Et si cette réalité n'est pas nouvelle, elle est d'autant plus prégnante qu'elle est toujours à l'origine de l'engagement des jeunes à entamer une formation longue et exigeante pour devenir musicien.

Nous ne pouvons guère résister à citer assez longuement le témoignage de Frank Leard qui, dans son livre L'univers des musiciens. Analyse d'une expérience artistique, pose un regard interrogateur sur la singularité du métier de musicien :

Nous jouions depuis plus d'une heure quand il est entré. L'étonnement qui se lisait dans son regard attira le mien. Je restai interloqué par sa stupeur. C'était comme s'il voyait pour la première fois un groupe de musiciens [...] Je me retournai vers mes compagnons de jeu pour m'assurer de la normalité de la situation. Le batteur semblait à son aise, le guitariste avait l'air d'être également à son affaire et la chanteuse s'efforçait de " chauffer » le public en produisant quelques vocalises de haut vol. Tout se passait comme d'habitude, rien qui puisse susciter une telle stupéfaction. Mais celle-ci perdurait et le jeune homme restait planté là, la bouche ouverte, comme s'il avait été en face d'une révélation de quelconque nature que je ne pouvais interpréter. Devant l'insistance du regard, je me retournai à nouveau, et c'est à cet instant que je fus saisi par l'étrangeté de la situation. Qu'étions-nous en train de faire exactement? Que faisions-nous là, nous autres musiciens, à jouer de la musique des années soixante-dix pour un public, qui, sans être indifférent, n'éprouvait pas d'enthousiasme particulier? À quoi servaient au juste toutes ces années de pratiques instrumentales, ces heures innombrables passées en répétition à se hasarder à concrétiser quelque chose que nous appelions notre rêve et dont nous avions nous-mêmes du mal à saisir les contours ou même à discourir sur nos véritables intentions (Leard 2008, p. 9-10).

La question de savoir à quoi peuvent servir «toutes ces années de pratiques instrumentales " nous semble une question primordiale dans un contexte où le statut du musicien et ce qu'il représente dans notre société évoluent à un rythme accéléré qui suit les transformations sociales, culturelles et technologiques de la société moderne. Elle rejoint aussi la constatation de Menger (2009, p. 224-236) qui relativise le poids des études dans l'accès au travail. Poser cette question lorsque l'on fait des études nous semble un excellent moyen non seulement de comprendre ces transformations en temps réel, mais aussi d'avoir un regard informé et critique sur le métier de musicien au-delà des définitions officielles de l'artiste, proposées par le Ministère de la culture, des communications et de la condition féminine du Québec et ses organismes affiliés comme le Conseil des arts et lettres du Québec ${ }^{5}$. Les travaux présentés dans ce numéro

5 Selon le CALQ, l'artiste professionnel qui, à ce titre peut bénéficier d'une bourse, se définit comme suit : 1) se déclare artiste professionnel ;2) crée des oeuvres ou pratique un art à son propre compte ou offre 
de la RMO posent principalement trois questions : 1) que veut dire devenir un musicien professionnel dans un contexte socioculturel en mutation constante ? ; 2) comment les études musicales servent-elles à cette intégration ?; et 3) de quelle manière se vit l'insertion professionnelle et sur quels dispositifs s'appuie-t-elle?

Les travaux présentés dans ce numéro de la RMO ne prétendent pas répondre à ces questions définitivement, d'autant que le Québec a fortement besoin d'un portrait de la profession musicale comme celui brossé par Coulangeon (2004) en ce qui concerne la France. Lesdits travaux se heurtent d'abord à la difficulté de définition même de ce qu'est un artiste. Rappelons que dans L'artiste, l'institution et le marché, Moulin se pose la question des critères à appliquer pour définir la profession d'artiste. S'agit-il d'indépendance économique, d'autodéfinition, de compétence spécifique ou de reconnaissance du milieu ? La réponse est un mélange de ces ingrédients savamment dosés qui donne malgré tout un résultat assez « flou » (Moulin [1992]1997, p. 249).

La définition du métier de musicien (interprètes et compositeurs) est d'autant plus floue ${ }^{6}$ qu'elle doit tenir compte des disparités immenses qui caractérisent le milieu musical et par conséquent des conditions primaires d'exercice du métier. Contrairement à d'autres métiers, le métier de musicien n'est pas régi par des règles corporatives et les conditions d'exercice varient au gré de l'économie générale du pays. Le résultat est celui d'une multiplicité de définition de tâches dans l'enveloppe étiquetée "musicien ». Être musicien, c'est jouer de la musique, composer de la musique, parler de musique, enseigner la musique, à temps plein - dans ce cas - la définition est plus solide, et à temps partiel - la situation est alors beaucoup plus "floue ». L'exercice du métier à temps partiel contribue grandement à la difficulté de la reconnaissance du statut d'artiste par rapport à d'autres professions puisque l'artiste, le musicien en l'occurrence, tire une partie, voire une grande partie de ses revenus d'une autre activité.

Les travaux publiés ici lèvent le voile sur cette situation, qui n'est pas propre au Québec d'ailleurs. Surtout, ils pavent la voie aux études qu'il reste à réaliser pour mieux comprendre la professionnalisation musicale ainsi que les différentes réalités de participation au marché de l'emploi musical propres au Québec.

ses services, moyennant rémunération, à titre de créateur ou d'interprète, notamment dans les domaines sous la responsabilité du Conseil des arts et des lettres du Québec ; 3) a une reconnaissance de ses pairs ; et 4) diffuse ou interprète publiquement des oeuvres dans des lieux et/ou un contexte reconnus par les pairs.

6 On peut par exemple se référer à la définition proposée par l'Unesco et citée par Moulin : « Toute personne qui considère sa création artistique comme un élément essentiel de sa vie, qui ainsi contribue au développement de l'art et de la culture, et qui est reconnue ou cherche à être reconnue en tant qu'artiste, qu'elle soit liée ou non par une relation de travail ou d'association quelconque (Recommendation Concerning the Status of the Artist, Paris, Unesco, 1980, p. 5 ; cité dans Moulin [1992]1997, p. 265). » 
viii Michel Duchesneau

ET DANick TROTtIER

BIBLIOGRAPHIE

Becker, Howard S. ([1988]2010), Les mondes de l'art, Paris, Flammarion.

Coulangeon, Philippe (2004), Les musiciens interprètes en France. Portrait d'une profession, Paris, Jacqueline Boucherat.

Freidson, Eliot (1986), "Les professions artistiques comme défi à l'analyse sociologique ", Revue française de sociologie, vol. 27, $\mathrm{n}^{\circ} 3$, p. 431-443.

Moulin, Raymonde ([1992]1997), L'artiste, l'institution et le marché, Paris, Flammarion.

Menger, Pierre-Michel (2009), Le travail créateur. S'accomplir dans l'incertain, Paris, Seuil/Gallimard.

Leard, Franck (2008), L'univers des musiciens. Analyse d'une expérience artistique, Toulouse, Presses universitaires du Mirail.

Articles

Le modèle, une voie vers la professionnalisation. L'exemple de Yuli Turovsky et de ses étudiants

1 Florence Leyssieux

Les paramètres de l'insertion professionnelle des musiciens au Québec. L'exemple de l'ensemble Magnitude6

20 Marie-Pier Leduc

Le cas Constantinople. Historique, identité musicale et enjeux de la professionnalisation des musiciens migrants

36 Caroline Marcoux-Gendron

Une synthèse de la littérature portant sur les enjeux philosophiques liés à l'intégration des Tic en éducation musicale

57 Jean-Philippe Després et Francis Dubé

Stratégies pédagogiques visant le développement des habiletés métacognitives du musicien en formation afin d'optimiser l'efficacité de ses pratiques instrumentales

74 Malinalli Peral Garcia et Francis Dubé

CONTRIBUTION LIBRE

La nasalisation dans la chanson country-western. Un cas de phonostylistique

90 Catherine Lefrançois 